\title{
Geometric scaling as traveling waves
}

\author{
S. Munier*t and R. Peschansk \\ Service de physique théorique, CEA/Saclay, 91191 Gif-sur-Yvette cedex, France ${ }^{\S}$
}

\begin{abstract}
We show the relevance of the nonlinear Fisher and Kolmogorov-Petrovsky-Piscounov (KPP) equation to the problem of high energy evolution of the QCD amplitudes. We explain how the traveling wave solutions of this equation are related to geometric scaling, a phenomenon observed in deepinelastic scattering experiments. Geometric scaling is for the first time shown to result from an exact solution of nonlinear QCD evolution equations. Using general results on the KPP equation, we compute the velocity of the wave front, which gives the full high energy dependence of the saturation scale.
\end{abstract}

1. Geometric scaling [1] is an interesting phenomenological feature of high energy deep-inelastic scattering (DIS). It is expressed as a scaling property of the virtual photon-proton cross section, namely

$$
\sigma^{\gamma^{*}}(Y, Q)=\sigma^{\gamma^{*}}\left(\frac{Q^{2}}{Q_{s}^{2}(Y)}\right)
$$

where $Q$ is the virtuality of the photon, $Y$ the total rapidity and $Q_{s}$ an increasing function of $Y$ called the saturation scale [2].

It is convenient to work within the QCD dipole picture of DIS 3]. In the leading $\operatorname{logs}(Y)$ approximation of perturbative QCD, the cross section factorizes as

$$
\sigma^{\gamma^{*}}(Y, Q)=\int_{0}^{\infty} x_{01} d x_{01} \int_{0}^{1} d z\left|\psi\left(z, x_{01} Q\right)\right|^{2} N\left(Y, x_{01}\right)
$$

$\psi\left(z, x_{01} Q\right)$ is the photon wave function on a $q \bar{q}$ dipole of size $x_{01}$, and $z$ is the longitudinal momentum fraction of the photon carried by the quark. $N\left(Y, x_{01}\right)$ is the dipole forward scattering amplitude. Let us define

$$
\mathcal{N}(Y, k)=\int_{0}^{\infty} \frac{d x_{01}}{x_{01}} J_{0}\left(k x_{01}\right) N\left(Y, x_{01}\right) .
$$

In this picture, the geometric scaling property reads

$$
\mathcal{N}(Y, k)=\mathcal{N}\left(\frac{k^{2}}{Q_{s}^{2}(Y)}\right) .
$$

Within suitable approximations (large $N_{c}$, summation of fan diagrams, spatial homogeneity) and starting from the Balitsky-Kovchegov equation [4], it has been shown [5] that this quantity obeys the nonlinear evolution equation

$$
\partial_{Y} \mathcal{N}=\bar{\alpha} \chi\left(-\partial_{L}\right) \mathcal{N}-\bar{\alpha} \mathcal{N}^{2}
$$

where $\bar{\alpha}=\alpha_{s} N_{c} / \pi, \chi(\gamma)=2 \psi(1)-\psi(\gamma)-\psi(1-\gamma)$ is the characteristic function of the BFKL kernel $\left.\underline{\underline{6}}\right], L=\log \left(k^{2} / k_{0}^{2}\right)$ and $k_{0}$ is some fixed low momentum scale. It is well-known that the BFKL kernel can be expanded to second order around $\gamma=\frac{1}{2}$, if one sticks to the kinematical regime $8 \bar{\alpha} Y \gg L$. We expect this commonly used approximation to remain valid for the full nonlinear equation (5). The latter boils down to a parabolic nonlinear partial derivative equation:

$$
\partial_{Y} \mathcal{N}=\bar{\alpha} \bar{\chi}\left(-\partial_{L}\right) \mathcal{N}-\bar{\alpha} \mathcal{N}^{2}
$$

with

$$
\bar{\chi}\left(-\partial_{L}\right)=\chi\left(\frac{1}{2}\right)+\frac{\chi^{\prime \prime}\left(\frac{1}{2}\right)}{2}\left(\partial_{L}+\frac{1}{2}\right)^{2} .
$$

\footnotetext{
* Permanent address after October, 1: Centre de physique théorique, École polytechnique, 91128 Palaiseau cedex, France.

$\S$ URA 2306, unité de recherche associée au CNRS.
} 
The question we want to address is whether there are exact asymptotic (in $Y$ ) solutions of Eq.(6) exhibiting geometric scaling.

2. Introducing the notation $\omega=\chi\left(\frac{1}{2}\right), D=\chi^{\prime \prime}\left(\frac{1}{2}\right)$, and defining $\bar{\gamma}=1-\frac{1}{2} \sqrt{1+8 \omega / D}$, the change of variables

$$
\begin{aligned}
t & =\frac{\bar{\alpha} D}{2}(1-\bar{\gamma})^{2} Y \quad, \quad x=(1-\bar{\gamma})\left(L+\frac{\bar{\alpha} D}{2} Y\right), \\
u(t, x) & =\frac{2}{D(1-\bar{\gamma})^{2}} \mathcal{N}\left(\frac{2 t}{\bar{\alpha} D(1-\bar{\gamma})^{2}}, \frac{x}{1-\bar{\gamma}}-\frac{t}{(1-\bar{\gamma})^{2}}\right)
\end{aligned}
$$

brings Eq.(6) for $\mathcal{N}$ into the Fisher and Kolmogorov-Petrovsky-Piscounov (KPP) equation [] for $u$ :

$$
\partial_{t} u(t, x)=\partial_{x}^{2} u(t, x)+u(t, x)(1-u(t, x)) .
$$

We are going to use some general results on the existence of traveling wave solutions to the KPP equation [8]. If one chooses an initial condition at time $t=t_{0}$ such that $u\left(t_{0}, x\right)$ decreases smoothly from 1 to 0 as $x$ goes from $-\infty$ to $+\infty$, and has the asymptotic behavior

$$
u\left(t_{0}, x\right) \underset{x \rightarrow+\infty}{\sim} e^{-\beta x},
$$

it is proven [8] that the KPP equation admits traveling wave solutions at large times. This means that there exists a function of one variable $w$ such that

$$
u(t, x) \underset{t \rightarrow+\infty}{\sim} w\left(x-m_{\beta}(t)\right)
$$

uniformly in $x$. Such a solution is depicted on Fig.(11). The function $m_{\beta}(t)$ depends on the initial condition:

$$
\begin{array}{ll}
m_{\beta}(t)=c(\beta) t+\mathcal{O}(1) & \text { for } \beta<\beta_{c}, \\
m_{\beta}(t)=2 t-\frac{1}{2} \log t+\mathcal{O}(1) & \text { for } \beta=\beta_{c}, \\
m_{\beta}(t)=2 t-\frac{3}{2} \log t+\mathcal{O}(1) & \text { for } \beta>\beta_{c},
\end{array}
$$

where $c(\beta)=\beta+1 / \beta$, and the critical value $\beta_{c}=1$ corresponds to the minimum of $c(\beta)$. Note that for critical or supercritical $\beta \geq \beta_{c}$, the velocity of the traveling wave front $d m_{\beta}(t) / d t$ is independent of $\beta$ up to a constant.

3. Let us show that the initial conditions for QCD are of the form of Eq. (10). For an initial value $Y=Y_{0}$, corresponding to $t=t_{0}=\bar{\alpha} D(1-\bar{\gamma})^{2} Y_{0} / 2$ and for large $L$, corresponding to large $x$, the behaviour of $\mathcal{N}$ is given by perturbative QCD. Asymptotically in $L$ (i.e. in $x$ at fixed $t$ ), it reads

$$
\mathcal{N} \propto k^{-2 \gamma_{0}}, \gamma_{0}=1
$$

We get the relationship between $\beta$ and $\gamma$ from the coordinate map (8): $\beta=\gamma_{0} /(1-\bar{\gamma})$. In particular, $u\left(t_{0},+\infty\right) \propto$ $\mathcal{N}\left(Y_{0}, L \rightarrow+\infty\right)=0$. Formula (13) through the Fourier transform (31), corresponds to QCD color transparency, namely $N\left(Y, x_{01}\right) \propto x_{01}^{2}$.

The condition $u\left(t_{0},-\infty\right)=1$ corresponds to the normalization condition $\mathcal{N}\left(Y_{0}, L \rightarrow-\infty\right)=D(1-\bar{\gamma})^{2} / 2$ when $k / k_{0} \rightarrow 0$. This infrared limit is not attainable in perturbative QCD, but it is reasonable to expect that some upper bound on $\mathcal{N}$ exist because of strong absorption.

Interestingly enough, $\beta$ happens to be equal to $2 / \sqrt{1+8 \omega / D}=1.55276 \ldots$, i.e. it lies within the supercritical regime $\beta>\beta_{c}$ of the KPP equation. Note that in this regime, possible logarithmic prefactors in Eq.(13) would not modify the traveling wave solution, at variance with the critical case $\beta=\beta_{c}$ [8]. Hence color transparency is the only relevant property for defining the initial condition at large $L$.

According to the above-mentioned mathematical framework, traveling wave solutions to Eq.(6) for $\mathcal{N}$ exist at large $Y$. We shall show that they correspond to specific geometric scaling solutions. Indeed, the velocity of the traveling wave front is given by the third equation in (12) because we are in the supercritical regime. This means that the large time solutions depend on a single variable $x-2 t+\frac{3}{2} \log t$. From the coordinate mapping (8), one sees that we may write the solution as

$$
\mathcal{N}(Y, k)=\mathcal{N}\left(\frac{k^{2}}{Q_{s}^{2}(Y)}\right) \quad \text { where } \quad Q_{s}^{2}(Y)=k_{0}^{2} Y^{-\frac{3}{2(1-\bar{\gamma})}} e^{\bar{\alpha} D\left(\frac{1}{2}-\bar{\gamma}\right) Y}
$$




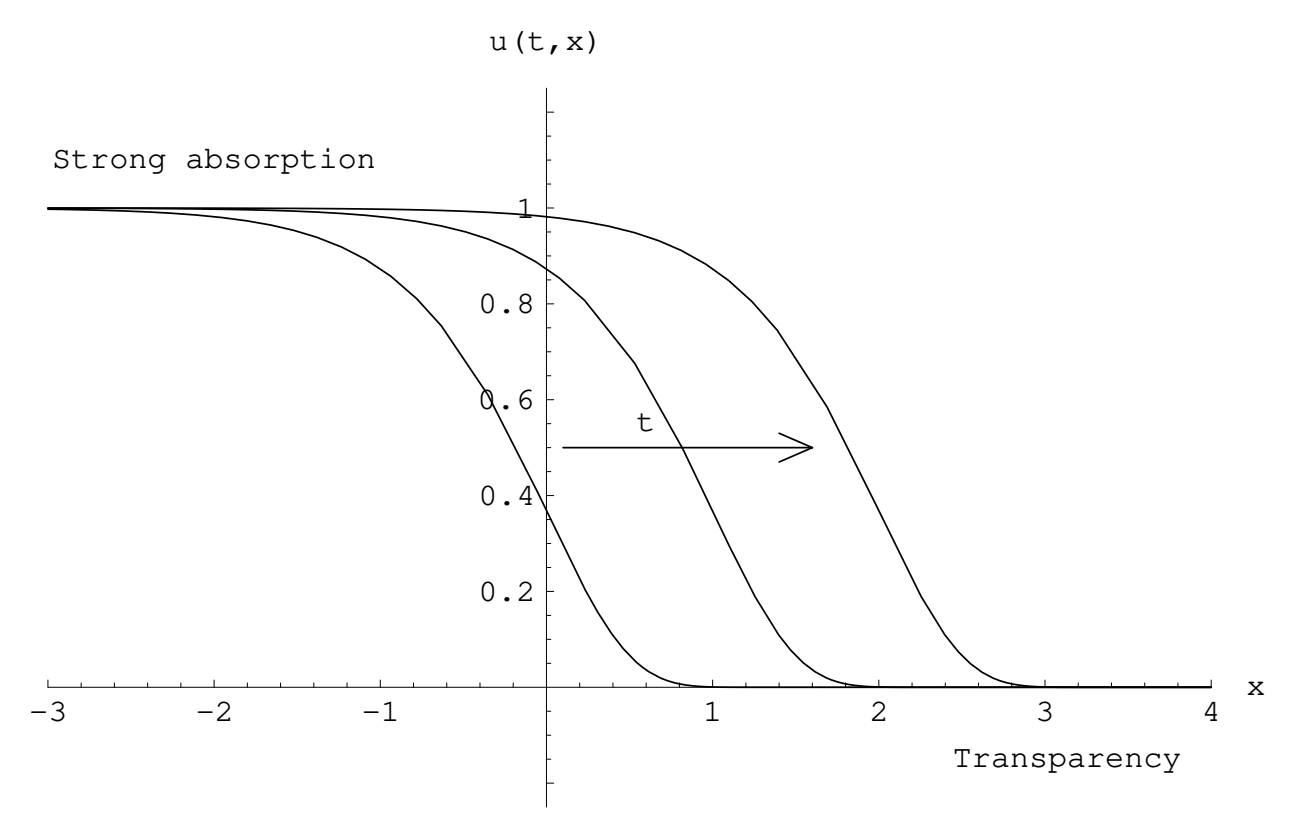

FIG. 1: Typical traveling wave solution. The function $u(t, x)$ is represented for three different times. The wave front connecting the regions $u=1$ and $u=0$ travels from the left to the right as $t$ increases. That illustrates how the "strong absorption" region invades the "transparency" region (see text).

We have absorbed all $\mathcal{O}(1)$ constants into $k_{0}^{2}$. Eq. (14) is nothing else than geometric scaling, $Q_{s}(Y)$ being the saturation scale, $c f$. Eq.(4).

4. Equation (14) is the exact asymptotic solution of the nonlinear Eq.(6) and thus geometric scaling appears as a universal property of this kind of equations. We obtain the saturation scale including the complete prefactor up to factors of order $\mathcal{O}(1)$. It comes from a non-trivial mathematical property of nonlinear equations. To our knowledge, it is the first exact result taking into full account the nonlinearities of a QCD evolution equation (6).

Comparing our results to previous studies, the exponential growth of the saturation scale with the rapidity $Y$ matches the estimates obtained from the extrapolation of the solution of the linear BFKL equation up to the saturation scale [9]. The powerlike prefactor that we get and prove to be universal, matches the one obtained in Ref. [10], where absorption was treated as a boundary condition on the linear equation [11].

Several numerical studies of Eq.(15) have been performed. Evidence for "soliton-like" solutions [12] and for geometric scaling has been found [12, 13]. Recently, formula (14) was proved to be very well verified [14]. This matches the mathematical properties we have discussed.

Among pending mathematical questions, it would be interesting to have a similar approach for the original equation (5), beyond the approximation (7). The question is whether geometric scaling is still valid in this case or if there are scaling violations which could also be universal. Another intriguing question is the meaning of the strong absorption bound in the initial condition. We think that the mathematical apparatus for nonlinear partial derivative equations could provide interesting hints for these questions.

As for the physical prospects of our approach, it is inspiring to note that the same equation appears in many different contexts, among which the problem of polymers on disordered trees [15]. In that context, $\beta$ plays the rôle of a temperature and there is a phase transition at $\beta=\beta_{c}$. $\beta>\beta_{c}$ corresponds to a phase of spin glass type. Similar features have been discussed in multiparticle collisions [16].

R.P. wishes to thank B. Derrida for introducing the subject of traveling waves to him, and E. Iancu for interesting discussions on saturation. 
† Electronic address: Stephane.Munier@cpht.polytechnique.fr

\# Electronic address: pesch@spht.saclay.cea.fr

[1] A. M. Staśto, K. Golec-Biernat, and J. Kwiecinski, Phys. Rev. Lett. 86, 596 (2001), hep-ph/0007192

[2] For a review on saturation and references, see A.H. Mueller, "Parton saturation: An overview", hep-ph/0111244

[3] N. N. Nikolaev and B. G. Zakharov, Z. Phys. C49, 607 (1991); A. H. Mueller, Nucl. Phys. B415, 373 (1994).

[4] I. I. Balitsky, Nucl. Phys. B 463, 99 (1996) arXiv:hep-ph/9509348; Y. V. Kovchegov, Phys. Rev. D60, 034008 (1999), hep-ph/9901281

[5] Y. V. Kovchegov, Phys. Rev. D61, 074018 (2000), hep-ph/9905214

[6] L. N. Lipatov, Sov. J. Nucl. Phys. 23, 338 (1976); E. A. Kuraev, L. N. Lipatov, and V. S. Fadin, Sov. Phys. JETP 45, 199 (1977); I. I. Balitsky and L. N. Lipatov, Sov. J. Nucl. Phys. 28, 822 (1978).

[7] R. A. Fisher, Ann. Eugenics 7, 355 (1937); A. Kolmogorov, I. Petrovsky, and N. Piscounov, Moscou Univ. Bull. Math. A1, 1 (1937).

[8] M. Bramson, Memoirs of the American Mathematical Society 285 (1983).

[9] E. Iancu, K. Itakura and L. McLerran, Nucl. Phys. A708, 327 (2002), hep-ph/0203137

[10] A. H. Mueller and D. N. Triantafyllopoulos, Nucl. Phys. B640, 331 (2002), hep-ph/0205167

[11] To see that the results coincide, one notes that $\bar{\gamma}$ is the solution of the equation $\chi^{\prime}(\gamma) / \chi(\gamma)=1 /(\gamma-1)$ when $\chi(\gamma)$ is approximated by $\bar{\chi}(\gamma), c f$. Eq.(17).

[12] M. Braun, Eur. Phys. J. C 16, 337 (2000) arXiv:hep-ph/0001268.

[13] E. Levin and K. Tuchin, Nucl. Phys. B 573, 833 (2000) arXiv:hep-ph/9908317; Nucl. Phys. A 691, 779 (2001) arXiv:hep-ph/0012167; N. Armesto and M. A. Braun, Eur. Phys. J. C 20, 517 (2001) arXiv:hep-ph/0104038; M. Lublinsky, Eur. Phys. J. C 21, 513 (2001) arXiv:hep-ph/0106112; K. Golec-Biernat, L. Motyka and A. M. Stasto, Phys. Rev. D 65, 074037 (2002) arXiv:hep-ph/0110325.

[14] J. L. Albacete, N. Armesto, A. Kovner, C. A. Salgado and U. A. Wiedemann, arXiv:hep-ph/0307179

[15] B. Derrida and H. Spohn, J. Stat. Phys. 51, 817 (1988).

[16] A. Bialas and R. Peschanski, Phys. Lett. B 207, 59 (1988); R. Peschanski, Nucl. Phys. B 327, 144 (1989); P. Brax and R. Peschanski, Nucl. Phys. B 353, 165 (1991). 